
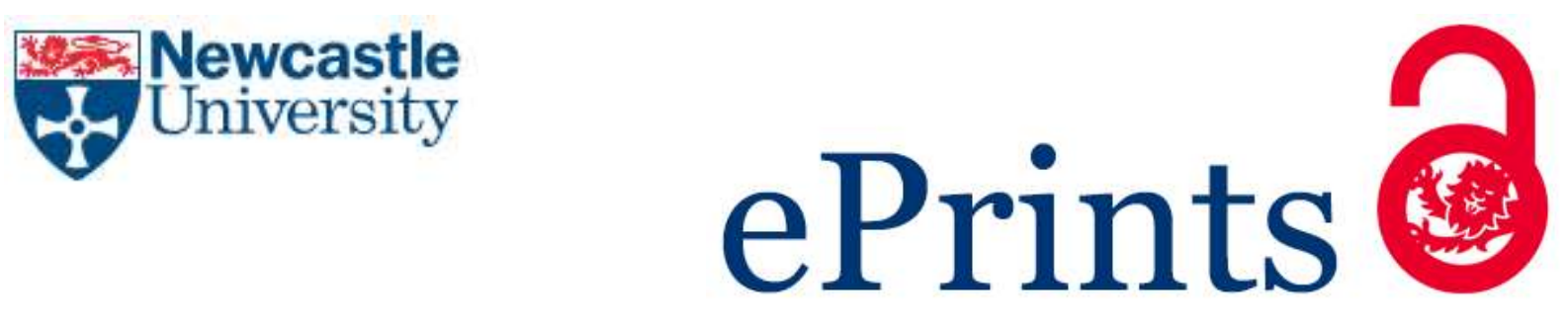

Gillivan-Murphy P, Miller N, Carding P. Voice Tremor in Parkinson's Disease: An Acoustic Study. Journal of Voice 2018

\title{
Copyright:
}

(C) 2018. This manuscript version is made available under the CC-BY-NC-ND 4.0 license

DOI link to article:

https://doi.org/10.1016/i.jvoice.2017.12.010

Date deposited:

$21 / 02 / 2018$

Embargo release date:

03 February 2019

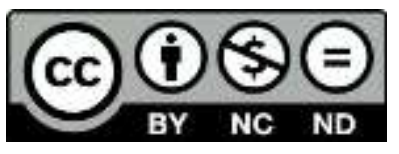

This work is licensed under a

Creative Commons Attribution-NonCommercial-NoDerivatives 4.0 International licence 


\section{Voice tremor in Parkinson's disease: an acoustic study}

\section{Authors:}

Patricia Gillivan-Murphy, PhD.

Speech \& Language Therapy Department, Mater Misericordiae University Hospital,

Eccles Street,

Dublin 7,

Ireland.

Nick Miller

Newcastle University Institute for Ageing

Speech and Language Sciences

George VI Building

Newcastle University

Newcastle-Tyne NE1 7RU

Great Britain

Paul Carding

Professor of Speech Pathology,

School of Allied and Public Health I Faculty of Health Sciences I Australian Catholic University

Based at: Brisbane Campus, Room 1 MCA Building

1100 Nudgee Road Banyo, Qld 4014

\section{Corresponding author}

Patricia Gillivan-Murphy, PhD.

Speech \& Language Therapy Department, Mater Misericordiae University Hospital,

Eccles Street,

Dublin 7,

Ireland.

pgmurphy@mater.ie

\section{Introduction}

Parkinson's disease (PD) is strongly associated with phonatory dysfunction ${ }^{12}$ and voicerelated disability ${ }^{3-5}$. Tremor is one of the classic triad of motor symptoms in PD alongside rigidity and bradykinesia, and is a commonly described feature of voice quality in PD.

Pathological voice tremor (i.e when associated with neurological disease) occurs when there is involuntary and rhythmical oscillatory movement in the vocal tract which causes rhythmic fluctuations in the fundamental frequency and amplitude of the voice ${ }^{67}$. These fluctuations are perceived as rhythmic (quasi-rhythmic) fluctuations in pitch and loudness.

It is unclear how many people with PD have a clinically identified voice tremor. Auditory perceptual studies have reported a prevalence range of $13 \%$ to $68 \%{ }^{89}$. Visual (endoscopic) 
studies that identify tremor behaviour have suggested a prevalence rate which varies from $14.6 \%$ to $55 \%{ }^{10}{ }^{11}$. One source of confusion is the varied terminology used in the literature: 'tremorous voice' 12 ; 'tremulousness' ${ }^{8}$; tremulous pitch (deficit in pitch steadiness)' 9 ; ' $p$ itch unsteadiness'13; ' 'perceptible vocal tremor'12; 'flutter' 14; 'vertical laryngeal tremor 11; 'laryngeal tremor' $15 ;$; 'rhythmic amplitude tremor'12. Further issues include the variance of the tasks used to elicit the tremor behaviour, and the lack of reported reliability of ratings ${ }^{5}$. Signal processing of the acoustic waveform may provide an accurate means of measuring voice tremor.

The acoustic waveform analysis has the potential to reflect the quasi-rhythmical movement of muscles in the vocal tract associated with voice tremor. The effect of oscillatory movement on the length of the vibratory cycle (frequency tremor) and/or the amplitude of the cycle (amplitude tremor) ${ }^{16}$ can be measured in addition to the rate of the tremor $(\mathrm{Hz})$.

Studies have employed acoustic measurement to differentiate patients with neurological disease from healthy controls ${ }^{16}$ and to differentiate between different neurological groups ${ }^{17}$. Several previous studies have applied acoustic analysis to the study of voice tremor in people with PD (pwPD) 12181920 . Two studies examined the presence of amplitude tremor ${ }^{12}$ ${ }^{18}$ in pwPD. Ramig et al., ${ }^{12}$ described a 'rhythmic amplitude tremor imposed upon cycle-to cycle deviancies', visually evident in the waveform of one of nine PD patients, which differed from the waveform of the patients with Myotonic Dystrophy, and Huntington's disease.

Stewart et al., ${ }^{18}$ visually identified (using narrow band spectrographic analysis), fluctuations in both amplitude and frequency tremor aspects in 33\% of a group of twelve patients with 'early' PD. Zarzur et al., ${ }^{19}$ visually identified tremor in spectrogram tracings of VOXMETRIA $®$ and GRAM $\AA$ in $69.5 \%$ of a group of twenty-six pwPD. Across these studies, there was no quantification of the amplitude and/or frequency tremor, or comparisons to healthy controls. In contrast, Tanaka et al., ${ }^{20}$ compared male and female pwPD with age-matched healthy controls on a range of acoustic voice measures including tremor parameters using the MultiDimensional Voice Programme (MDVP) from the Computerised Speech Laboratory (CSL). They found that frequency tremor intensity index (FTRI) \% and frequency tremor frequency (Fftr) $\mathrm{Hz}$ was significantly higher in male PD relative to controls, and that FTRI (\%) only differentiated female PD from controls. It is not clear from the study if findings are based on one trial of a sustained /a/ vowel or on a number of trials.

Acoustic analysis of the waveform has significant potential value to enable an improved understanding of voice tremor in pwPD and help determine if there are differences between pwPD and neurologically healthy age-matched controls. The current literature is limited and has no clear conclusions regarding the characteristics of frequency and amplitude tremor in 
PD due to a combination of: a small sample size; lack of a control group; a heterogenous grouping of patients; the paucity of detailed information relating to the methods used. Therefore the primary aim of the current study is to determine if acoustic voice tremor analysis differentiates pwPD from neurologically healthy controls, and to describe tremor characteristics in PwPD. A secondary aim is to place the acoustic voice tremor findings in the broader context of voice disability and disease variables.

\section{Methods}

Participants

Ethical approval for the study was granted by the Ethics committee of the Mater Misericordiae University Hospital (MMUH). Two groups were recruited for the study: patients with idiopathic Parkinson's disease (PD group) and neurologically healthy controls (control group).

\section{PD group}

Consecutive patients attending a routine hospital movement disorders outpatient clinic with a diagnosis of PD ${ }^{21}$ made by a consultant neurologist specialist in PD were screened for eligibility to take part. Presence of perceived voice tremor was not a requirement for entry inclusion. The following exclusion criteria were applied: a concomitant neurological disease; dementia [score $\leq 23$ on the Mini-Mental State Examination (MMSE)] ${ }^{22}$; a psychological/psychiatric disorder; taking tremor inducing medication (lithium, anticonvulsant medication, immune-suppressants, bronchodilators); a current smoker or an ex-smoker for less than five years; a history of cancer of the head and neck region, speech or voice problems unrelated to and prior to the onset of PD; receiving speech/voice treatment or received Lee Silverman Voice Treatment (LSVT) in the previous two years; reported inadequate hearing (unable to hear conversational speech comfortably), and/or wearing hearing aids; unable to postpone dopaminergic medication for twelve hours in advance of study testing, and unable to fast for food and liquids (excepting water) on the morning that testing was carried out; non-native English speaker; presence of dyskinesias severe enough to contra indicate vocal tract examinations (patients had a range of assessments carried out including nasendoscopy for visual perceptual rating of tremor). 


\section{Control group}

A healthy neurological age-and-sex matched group was recruited. The same inclusion and exclusion criteria applied as to the PD group. Controls were recruited by inviting family members of PD participants, staff at the hospital, and parents of colleagues to get involved in the study.

\section{General procedures}

PwPD on a dopaminergic medication regime were tested in a practically defined "off" medication state, i.e., after a 12-hour overnight withdrawal of anti-parkinsonian medication ${ }^{23}$. All PD and control participants were evaluated in the morning between 08.00am and 10.00am in a single visit to to the hospital.

Disease duration was measured in years from the time of neurological diagnosis. PD overall severity was evaluated using the patient- derived questionnaire of Activities of Daily Living (part II) and the clinician-derived test of motor function (part III) from the Unified Parkinson's Disease rating Scale (UPDRS ) ${ }^{21}$. Possible scores for UPDRS II range from 0 (unaffected) to 52 and 0 (unaffected) to 108 for UPDRS III.

A range of assessments were carried out as part of a larger study on voice tremor in PD. In addition to UPDRS testing, auditory perceptual speech and voice measures and nasendoscopy were carried out. Patients and controls completed the Voice Handicap Index for patient self-rated voice disability ${ }^{24}$.

\section{Voice recordings and data preparation}

Voice recordings were carried out in a sound treated room with ambient noise levels measured at $50 \mathrm{~dB}$ sound pressure level (SPL). An AKG-C420 head-mounted microphone connected to the CSL was placed on the participant's head and placed $10 \mathrm{cms}$ from the angle of the mouth. A sustained /a/ vowel of 4.5 second duration was recorded directly on to the CSL, at a sampling rate of $50,000 \mathrm{~Hz}$. Training trials were carried out prior to the test trials to ensure participants understood the task, and that any technical problems that might arise could be rectified in advance of the test trials. Participants were instructed to sustain the vowel /a/ at a comfortable pitch and loudness. The Voice \& Tremor Protocol from CSL provides an example of a sustained /a/ vowel audio signal with additional visual wave form from a male voice ( 5 second duration). The participants were asked to listen to the registered 
voice from CSL, and then repeat the task. For the test trials, each participant sustained the vowel /a/ three times and each recording was saved to the Kay data file for later analysis.

The recordings for each participant were trimmed to 3 seconds (the initial 1 second and final 0.5 second segment of the signal was removed) and re-digitised. Consistent with other studies, the value of 3 seconds was chosen since it was considered to be sufficiently long to afford reliable analysis ${ }^{25} 26$. The trimmed voice signals for the PD and control groups were used for acoustic analyses.

\section{Acoustic analyses}

The Voice and Tremor Protocol (VTP) from the Motor Speech Profile (Advanced), a module of the CSL, Model 5141 from Kay Pentax (Lincoln Park, NJ), was used for the analysis ${ }^{27}$. The VTP yields a range of nine to thirteen voice and tremor related parameters, based on a sustained /a/ vowel task. An important aspect of measuring and classifying tremor is identifying the rate measured in cycles per second $(\mathrm{Hz})$, the regularity or periodicity, and the extent or magnitude. Involuntary movement (non-periodic) may be present in the vocal tract and is distinct from tremor. It is important to capture this phenomenon also with long term measures of instability which are sensitive to the detection of non-periodic tremor.

For this study, the rate, periodicity, and magnitude of frequency and amplitude tremor were selected from the VTP as the key tremor measures for further analyses. The rate of tremor refers to the rate of modulation of fundamental frequency and/or amplitude in the voice signal, measured in cycles per second $(\mathrm{Hz})$. In order for the rate of tremor to be determined the voice tremor has to have a certain level of regularity. Periodicity (\%) is a measure of the regularity of the tremor. The higher the periodicity the more regular is the tremor. The magnitude (\%) of tremor (frequency and amplitude) is a measure of the extent of variation in frequency and/or amplitude secondary to the effect of involuntary movement in the muscles of the vocal tract. Adjunctive measures of unsteadiness were also included. The selected VTP measures for the study are outlined below and for the purpose of completeness descriptions of all the measure ${ }^{27}$ generated from the VTP are outlined in Appendix 1.

\section{Key tremor measures}

- Rate of frequency tremor $[\mathrm{Rftr}(\mathrm{Hz})]$

- Rate of amplitude tremor [Ratr $(\mathrm{Hz})]$

- Periodicity of frequency tremor [Pftr (\%)] 
- Periodicity of amplitude tremor [Patr (\%)]

- Magnitude of frequency tremor [Mftr (\%)]

- Magnitude of amplitude tremor [Matr (\%)]

\section{. Adjunctive measures of unsteadiness}

- Coefficient of variations in the Fundamental Frequency [VFo)(\%)]

- Coefficient of variations in amplitude [vAm) (\%)]

\section{Data preparation}

The mean value across the three /a/ trials was calculated for each acoustic measure.

Total scores for UPDRS II and III were recorded separately. For the VHI, a summed total score was obtained. Additionally the scores from the physical, emotional and functional items of the VHI were also summed yielding three different subscale scores.

\section{Data analysis}

Statistical analysis was conducted in SPSS programme version 17.0 (Windows). Differences were considered significant at alpha level $<0.05$. Normality of distribution was determined for ordinal (UPDRS II and III, VHI) and for continuous data (acoustic measures) using the Kolmogoov-Smirnov test. To determine whether statistically significant differences existed between median sores of pwPD and control groups, a Mann Whitney U Test was applied to the acoustic measures and VHI scores. Spearman's rho was calculated to examine the relationship between acoustic voice tremor measures and disease duration, UPDRS II Activities of Daily Living, UPDRS III motor symptoms, and the VHI. When the acoustic tremor measures were plotted against disease duration, PD 5 emerged as an outlier. A review of the raw acoustic data showed wide variability of values across the three /a/ trials for Mftr, Matr, vFo and vAm, thus PD 5 was removed from the data pool for the correlational analysis. In the absence of prior studies that could provide reliable estimates of anticipated effect sizes for the chosen variables between people with and without PD, a post-hoc power analysis of the acoustic tremor measures obtained for this study was carried out. The effect sizes established for this study formed the basis of estimates of the power of the current study to detect differences given the sample sizes employed.

\section{Reliability}


Twelve (20\%) acoustic signal wave forms were independently re-digitised by a second researcher. For consistency's sake the first trial of each participant was chosen. Following redigitisation a comparison was made by the independent researcher with the values from the original acoustic signal to determine: if both researchers were consistent in the way they trimmed the data for analysis; that the values from the analysis had been correctly read off from the print out of the automatic analysis. Identical values were found for repeated analyses of the same segments, therefore no further measures of agreement were calculated. 


\section{Results}

Table 1 shows the descriptive statistics for gender, age, age at diagnosis, disease duration, UPDRS II and UPDRS III scores. There were no significant differences between PD and control groups by age or gender proportions, nor between gender groups according to age.

Table 1. Mean (SD), range values for gender, age, age at diagnosis, disease duration, UPDRS II, UPDRS III and $p$ values for PD and control group

\begin{tabular}{|l|l|l|}
\hline & $\begin{array}{l}\text { PD group } \\
(\mathrm{n}=30)\end{array}$ & $\begin{array}{l}\text { Control group } \\
(\mathrm{n}=28)\end{array}$ \\
$\begin{array}{l}\text { Males } \\
\text { Females }\end{array}$ & $\begin{array}{l}22(73 \%) \\
8(27 \%)\end{array}$ & $\begin{array}{l}20(71 \%) \\
8(29 \%)\end{array}$ \\
\hline Age (years) & & \\
\hline $\begin{array}{l}\text { Mean (SD) } \\
\text { Range }\end{array}$ & $\begin{array}{l}61.40(10.31) \\
(34-76)\end{array}$ & $\begin{array}{l}60.11(9.54) \\
(36-74)\end{array}$ \\
Males & $60.95(10.82)$ & $59.55(10.24)$ \\
Females & $62.63(9.29)$ & $61.50(7.96)$ \\
\hline $\begin{array}{l}\text { Age at diagnosis } \\
\text { Range }\end{array}$ & $56.17(9.56)$ & \\
\hline $\begin{array}{l}\text { Disease duration (yrs) } \\
\text { Range }\end{array}$ & $5.23(3.17)$ & \\
\hline UPDRS II & $(1-12)$ & \\
Range & $10.20(3.85)$ & \\
\hline $\begin{array}{l}\text { UPDRS III } \\
\text { Range }\end{array}$ & $(4-19)$ & \\
\hline
\end{tabular}




\section{Acoustic Tremor Measures}

Due to technical difficulties with the re-digitisation, seven of the pwPD group had data on two instead of three trials. For controls, three participants had data on two trials and one participant had data on one trial. For pwPD and control participants with two trials, the mean value of the two trials was calculated.

The detection rate for frequency and amplitude tremor together with tremor rate, periodicity, and magnitude, effect sizes and p-values for pwPD and control groups is shown in table 2 . The MSP gives values for tremor rate and periodicity only for those speakers where it detects periodic perturbation. Therefore data is based on the number in each group and condition where tremor was detected by MSP. Tremor was detected in PwPD and controls with similar frequency. For frequency tremor, pwPD had a higher rate, greater periodicity, and greater magnitude of tremor than controls. However the difference was not statistically significant. For amplitude tremor, pwPD had a statistically significant $(p=0.001)$ higher rate of amplitude tremor than controls. Periodicity of amplitude tremor was higher in pwPD than controls, and approached significance. Magnitude of tremor was higher in pwPD than controls without statistical significance. For co-efficient of variation in fundamental frequency, PwPD had a higher percentage than controls without statistical significance. For co-efficient of variation in amplitude, pwPD had a lower percentage than controls, though not statistically significant. Effect sizes (Cohen's $d$ ) for rate, periodicity and magnitude of frequency and amplitude tremor ranged from medium to large, with rate of amplitude tremor showing the largest effect size of 1.2. In contrast, effect sizes were smaller for long term measures of frequency (vFO\%) and amplitude (vAm\%) variation (Table 2). Power analysis yielded values of 0.79 , (Rftr Hz), 0.58 (Pftr\%), 0.40 (Mftr\%) and 0.29 (vF0\%) for frequency tremor differences between pwPD and controls. For amplitude tremor differences values of 0.999 (Ratr Hz), 0.86 (Patr\%), 0.71 (Matr \%), and 0.12 (vAm\%) were found. 
Table 2. Tremor detection rate [n(\%)], and mean (SD), range, median, and IQR values for tremor rate, periodicity, magnitude of tremor (frequency \& amplitude) and coefficient of variation (fundamental frequency, amplitude) for pwPD and control groups

\begin{tabular}{|c|c|c|c|c|c|c|}
\hline \multirow[b]{2}{*}{$\begin{array}{l}\text { Tremor detected by } \\
\text { MSP }\end{array}$} & \multicolumn{2}{|c|}{ Frequency Tremor } & \multirow[b]{2}{*}{$\begin{array}{l}\text { Effect } \\
\text { Size }\end{array}$} & \multicolumn{2}{|c|}{ Amplitude Tremor } & \multirow[b]{2}{*}{$\begin{array}{l}\text { Effect } \\
\text { size }\end{array}$} \\
\hline & PD & Control & & PD & Control & \\
\hline n (\%) & $13(43.3)$ & $10(35.7)$ & & $16(53.3)$ & $17(60.7)$ & \\
\hline \multicolumn{7}{|l|}{ Tremor rate $(\mathbf{H z})$} \\
\hline Mean (SD) & $4.39(2.59)$ & $3.03(1.33)$ & 0.661 & $4.94(2.25)$ & $2.85(0.72)$ & 1.251 \\
\hline Median & 3.25 & 2.66 & & 4.44 & 2.66 & \\
\hline IQR & $\begin{array}{l}2.43-6.54 \\
p=0.19\end{array}$ & $2.29-3.03$ & & $\begin{array}{l}3.40-5.69 \\
p=0.001\end{array}$ & $2.30-3.35$ & \\
\hline \multicolumn{7}{|l|}{ Periodicity \% } \\
\hline Mean (SD) & $30.22(16.50)$ & 24.09 (8.11) & 0.472 & $45.07(10.64)$ & $37.12(12.35)$ & 0.690 \\
\hline Median & 25.14 & 22.61 & & 43.40 & 38.84 & \\
\hline IQR & $\begin{array}{l}19.67-39.52 \\
p=0.38\end{array}$ & 18.44-27.53 & & $\begin{array}{l}34.21-55.39 \\
p=0.057\end{array}$ & $31.33-44.37$ & \\
\hline \multicolumn{7}{|l|}{ Magnitude \% } \\
\hline Mean (SD) & $0.91(0.73)$ & $0.71(0.12)$ & 0.382 & $3.23(2.08)$ & $2.27(0.91)$ & 0.598 \\
\hline Median & 0.69 & 0.68 & & 2.50 & 2.10 & \\
\hline
\end{tabular}




\begin{tabular}{|c|c|c|c|c|c|c|}
\hline IQR & $\begin{array}{l}0.40-0.69 \\
p=0.71\end{array}$ & $0.37-0.63$ & & $\begin{array}{l}1.49-3.11 \\
p=0.16\end{array}$ & $1.59-2.22$ & \\
\hline \multicolumn{3}{|c|}{ Variation of fundamental frequency (vFO\%) } & & \multicolumn{2}{|c|}{ Variation of amplitude (vAm \%) } & \\
\hline Mean (SD) & $1.58(2.5)$ & $1.06(0.5)$ & 0.288 & $7.86(3.43)$ & $8.26(3.03)$ & 0.124 \\
\hline Median & 1.06 & 0.93 & & 6.85 & 7.93 & \\
\hline \multirow[t]{2}{*}{ IQR } & $0.86-1.36$ & $0.71-1.22$ & & $5.98-8.49$ & $6.12-9.27$ & \\
\hline & \multicolumn{2}{|l|}{$p=0.164$} & & \multicolumn{2}{|l|}{$p=0.335$} & \\
\hline
\end{tabular}


Table 3 displays summary statistics for the VHI total and sub score results.

Table 3. Mean (SD), range, median, (IQR) and $p$ values for total and subscale VHI scores for PD and control group

\begin{tabular}{|c|c|c|c|c|c|}
\hline & $\begin{array}{l}\mathrm{PD}(\mathrm{n}=30) \\
\mathrm{M} 22, \mathrm{~F} 8\end{array}$ & $\begin{array}{l}\text { Control }(n=27) \\
\text { M19, F8 }\end{array}$ & $U$ & $Z$ & $p$-value \\
\hline \multicolumn{6}{|l|}{ Total VHI } \\
\hline Mean (SD) & $19.50(15.11)$ & $4.00(6.02)$ & 156.000 & -4.012 & $<0.001$ \\
\hline Range & $(0-49)$ & $(0-24)$ & & & \\
\hline Median (IQR) & $19.00(5.75-33.25)$ & $1.00(0.00-6.00)$ & & & \\
\hline \multicolumn{6}{|l|}{ Subtests } \\
\hline & & & & & \\
\hline \multicolumn{6}{|l|}{ Functional } \\
\hline Mean (SD) & $6.53(4.92)$ & $2.00(2.51)$ & 185.500 & -3.558 & $<0.001$ \\
\hline Range & $(0-16)$ & $(0-9)$ & & & \\
\hline Median (IQR) & $6.50(1.00-11.00)$ & $1(0.00-4.00)$ & & & \\
\hline \multicolumn{6}{|l|}{ Emotional } \\
\hline Mean (SD) & $4.97(5.16)$ & $0.67(2.13)$ & 179.000 & -4.025 & $<0.001$ \\
\hline Range & $(0-17)$ & $(0-10)$ & & & \\
\hline Median (IQR) & $3.50(0.00-9.00)$ & $0.00(0.00-0.00)$ & & & \\
\hline \multicolumn{6}{|l|}{ Physical } \\
\hline Mean (SD) & $8.00(6.16)$ & $1.33(2.34)$ & 164.500 & -3.964 & $<0.001$ \\
\hline Range & $(0-18)$ & $(0-10)$ & & & \\
\hline Median IQR & $9.50(0.75-13.00)$ & $0.00(0.00-2.00)$ & & & \\
\hline
\end{tabular}

Correlational analysis of tremor measures and VHI total and subscale scores showed no significant correlations, positive or negative for pwPD or controls (Table 4). 
Table 4. Spearman's (rho) for Rftr, Ratr, Mftr, Matr, vFo, vAm and VHI total and subscale scores, for PD and control group

\begin{tabular}{|c|c|c|c|c|c|c|c|c|}
\hline & \multicolumn{8}{|c|}{ VHI total and subscale scores } \\
\hline & \multicolumn{4}{|c|}{ PD group $(n=30)$} & \multicolumn{4}{|c|}{ Control group $(n=27)$} \\
\hline $\begin{array}{l}\text { Acoustic } \\
\text { Measures }\end{array}$ & Total & Functional & Physical & Emotional & Total & Functional & Physical & Emotional \\
\hline $\operatorname{Rftr}^{\mathrm{a}}(\mathrm{Hz})$ & 0.280 & 0.265 & 0.188 & 0.170 & -0.351 & -0.436 & -0.149 & -0.114 \\
\hline $\operatorname{Ratr}^{\mathrm{b}}(\mathrm{Hz})$ & -0.096 & 0.034 & -0.044 & -0.299 & -0.219 & -0.173 & -0.173 & -0.364 \\
\hline Mftr (\%) & 0.007 & -0.049 & 0.047 & -0.061 & 0.333 & 0.312 & 0.269 & 0.107 \\
\hline Matr (\%) & -0.107 & -0.150 & -0.011 & -0.123 & 0.213 & 0.186 & 0.185 & 0.049 \\
\hline vF0 (\%) & 0.052 & 0.085 & 0.028 & 0.026 & 0.145 & 0.161 & 0.078 & 0.112 \\
\hline vAm (\%) & -0.155 & -0.212 & -0.126 & -0.129 & 0.264 & 0.354 & -0.042 & 0.251 \\
\hline
\end{tabular}


Relationship between tremor measures, and disease variables, and age

Table 5 shows that the magnitude of frequency tremor was positively and significantly correlated with disease duration. To ensure that increasing age was not a contributory factor to results, further analysis was carried out to explore the relationship between voice tremor measures and age for pwPD and controls. The findings show that there was no relationship between acoustic measures and age for pwPD. There were positive significant relationships between acoustic measures and age for controls (Table 6).

Table 5. Spearman's correlation coefficient (rho) for Rftr, Ratr, Mftr, Matr, vFo, vAm, and disease duration with outlier (PD5) removed from the analysis

\begin{tabular}{|l|l|}
\hline Acoustic Measures & Disease duration \\
\hline Rate frequency tremor Rftr $(\mathrm{Hz})$ & $-0.147(\mathrm{n}=12)$ \\
\hline Rate amplitude tremor Ratr $(\mathrm{Hz})$ & $0.192(\mathrm{n}=15)$ \\
\hline Magnitude frequency tremor Mftr (\%) & $\mathbf{0 . 4 6 3 ^ { * }}$ \\
\hline Magnitude amplitude tremor Matr (\%) & 0.355 \\
\hline Variation in frequency vFo (\%) & 0.344 \\
\hline Variation in amplitude vAm (\%) & 0.202 \\
\hline${ }^{*}<0.05$ &
\end{tabular}

Table 6. Spearman's correlation coefficient (rho) for Rftr, Ratr, Mftr, Matr, vFo, vAm, and age (years) for PD and control group, with PD 5 excluded

\begin{tabular}{|l|l|l|}
\hline Acoustic measures & \multicolumn{2}{|c|}{ Age } \\
\hline & PD group $(\mathrm{n}=29)$ & Control group $(\mathrm{n}=28)$ \\
\hline & rho & rho \\
\hline Rate of frequency tremor Rftr $(\mathrm{Hz})$ & $0.014(\mathrm{n}=12)$ & $-0.401(\mathrm{n}=10)$ \\
\hline Rate of amplitude tremor Ratr (Hz) & $-0.284(\mathrm{n}=15)$ & $0.193(\mathrm{n}=17)$ \\
\hline $\begin{array}{l}\text { Magnitude of frequency tremor Mftr } \\
(\%)\end{array}$ & 0.1533 & $\mathbf{0 . 5 0 9}^{\star *}$ \\
\hline $\begin{array}{l}\text { Magnitude of amplitude tremor } \\
\text { Matr (\%) }\end{array}$ & -0.094 & $\mathbf{0 . 4 0 7}^{*}$ \\
\hline $\begin{array}{l}\text { Variation Fundamental Frequency } \\
\text { vFo (\%) }\end{array}$ & 0.103 & $\mathbf{0 . 5 2 8}{ }^{\star *}$ \\
\hline $\begin{array}{l}\text { Variation in Amplitude } \\
\text { vAm (\%) }\end{array}$ & 0.160 & 0.340 \\
\hline
\end{tabular}

${ }^{*}<0.05^{* *}<0.01$ : 
Table 7 displays the correlational findings for acoustic measures and total score for UPDRS II and III. Rate of amplitude tremor was negatively and significantly correlated with UPDRS III total score.

Table 7. Spearman's correlation coefficient (rho) for Rftr, Ratr, Mftr, Matr, vFo, Vam and UPDRS II, UPDRS III and UPDRS III tremor score

\begin{tabular}{|l|l|l|}
\hline & $\begin{array}{l}\text { UPDRS II total } \\
\text { score }\end{array}$ & $\begin{array}{l}\text { UPDR III total } \\
\text { score }\end{array}$ \\
\hline Acoustic measure & rho & rho \\
\hline Rate frequency tremor $(\mathrm{Hz})$ & $0.283(\mathrm{n}=13)$ & $0.168 \quad(\mathrm{n}=13)$ \\
\hline Rate amplitude tremor $(\mathrm{Hz})$ & $-0.425(\mathrm{n}=16)$ & $-0.509^{*}(\mathrm{n}=16)$ \\
\hline Magnitude frequency tremor $(\%)$ & -0.009 & 0.099 \\
\hline Magnitude amplitude tremor $(\%)$ & -0.018 & 0.299 \\
\hline Variation in frequency (\%) & -0.149 & 0.110 \\
\hline Variation in amplitude (\%) & -0.126 & 0.111 \\
\hline$* 0.05$ & & \\
\hline
\end{tabular}




\section{Discussion}

This is the first prospective study and the largest data set to report on acoustic voice tremor measures in pwPD and neurologically healthy controls. A primary aim of the study was to determine if there are differences between pwPD and neurologically health controls in relation to acoustic voice tremor measures. A secondary aim was to place the acoustic voice tremor findings in the broader context of voice disability and disease variables.

There were more males than females recruited into this study of thirty pwPD study, with a male to female ratio of 2.75:1. In comparison to other studies, the ratio of males to females in this study is both higher than the 1.5 male-to-female ratio reported by Midi et al., ${ }^{5}$ and lower than the 3.4 and 3.1 reported by Perez et al., ${ }^{11}$ and Goberman et al., ${ }^{28}$ respectively. The age profile [(mean 61 (SD 10) range 34-76 years)] of the pwPD in this study was very similar to D'Alatri et al., ${ }^{29}$ (mean 60 years), Stewart et al., ${ }^{18}$ (mean 59 years) and Perez et al's., ${ }^{11}$ study (mean 65 years). The PD group represented the milder end of the disease spectrum in relation to disability. Disease duration was relatively short with almost $40 \%$ receiving their PD diagnosis within the previous three years.

In the current study, frequency and amplitude tremor was detected by MSP in approximately $50 \%$ of pwPD. Amplitude tremor was detected in a slightly higher number of pwPD than frequency tremor. Other studies have reported the presence of frequency and amplitude tremor in a small sample of pwPD ${ }^{12}{ }^{18}$, on the basis of visual perception of tremor in a waveform. However this study goes further with a larger sample size and quantification of the acoustic tremor measures.

An important finding is that frequency and amplitude tremor was also detected in controls, and that amplitude tremor was detected in a similar number of pwPD and controls. The study findings therefore indicate that frequency and amplitude tremor is a feature of this group of pwPD, and of neurologically healthy controls. Boutsen et al., ${ }^{30}$ in a study of patients with ataxic dysarthria also reported detection of frequency and amplitude tremor in a comparison group of nineteen controls, using the same tremor protocol from MSP. However they reported lower rates of detection than the current study, and this is likely to be explained by the fact that control participants in the current study were older than those in Boutsen et al's., ${ }^{30}$ study. 
The rate of tremor is one of the parameters used in the classification of tremors with a $4-6 \mathrm{~Hz}$ tremor rate associated with rest tremor in $\mathrm{PD}^{31}$. In this study, the rate of frequency tremor did not differentiate pwPD from controls. However the rate of amplitude tremor differentiated the groups. It appears therefore that the rate of amplitude tremor may be one useful diagnostic indicator in relation to PD voice symptomatology ${ }^{30}$.

The measure of periodicity relates to the regularity of the detected tremor. The detected frequency and amplitude tremor was more periodic (closer to 100\%) in pwPD than in controls. Periodicity of frequency tremor varied much more in pwPD than in controls. This variability coupled with the small sample size may have resulted in the lack of statistical significance between the groups. The difference between the groups for periodicity of amplitude tremor approached significance.

The magnitude of frequency and amplitude tremor was greater in pwPD than controls. However it did not differentiate the groups. In relation to findings for pwPD, there were important similarities and differences between the present study findings and other studies. The magnitude of frequency tremor (median 0.69\%) for pwPD in the current study was very similar to D'Alatri et al's ${ }^{29}$ reported value $(0.63 \%)$ in their study of twelve pwPD. In contrast the magnitude of amplitude tremor (median $2.50 \%$ ) was lower in the present study than the value of $4.72 \%$ reported by D'Alatri et al. ${ }^{29}$ Both studies were similar with respect to acoustic analysis software analysis, mean age of participants, and data collection in an off-medication state. However there were important differences also between the studies which may explain the difference in findings. Firstly, D'Alatri et al's ${ }^{29}$ reported median values were based on a single sustained /a/ trial, whereas the current study was based on the mean of three trials for each participant. Secondly, the pwPD group had deep brain stimulation (DBS) carried out two to five years before the study evaluation was carried out. Finally, the participants in D'Alatri et al's., ${ }^{29}$ study had greater disease severity [higher scores on the Unified Parkinson's Disease Rating Scale (UPDRS) motor scale]. DBS is generally carried out on patients at the severe end of the disease spectrum, who are no longer benefitting from dopaminergic medication, and have significant tremor and/or dyskinesias. The mean disease duration for the present study was much lower at 5.23 years (range 1-12) versus 12.50 years (range 7-28) in D'Alatri et al's study.

In contrast to the present study findings, Jiang et al., ${ }^{17}$ reported that the 'magnitude of amplitude modulation' differentiated their 'pathological' tremor group $(n=10)$ from the normal control group. However caution is required regarding interpretation of their findings in the context of the current study for two reasons. Firstly, they had a heterogeneous study group, seven had PD and one had 'idiopathic tremor', (understood by this study's main author to be 
an 'essential tremor'). Diagnostic information was not provided on the remaining two participants. Secondly, their method of quantifying the magnitude of amplitude tremor was different to the current study, and to D'Alatri et al's. ${ }^{29}$ study. Jiang et al., ${ }^{17}$ applied Fast Fourier transform (FFT) to generate power spectra from a 2 second simultaneously recorded acoustic and airflow signal. They developed a peak detecting algorithm to identify spectral peaks below $30 \mathrm{~Hz}$ and then calculated the peak prominence ratio for each spectral peak using a formula.

Ancillary tremor measures of overall variation in frequency (vFo\%) and amplitude (vAm\%) were included in this study to broaden the scope of the analysis, and increase understanding of tremor measures. The findings show that pwPD had a greater amount of overall frequency variation (vFo) than controls, though the difference was not significant. An unexpected finding was the greater amount of variations in amplitude (vAm\%) in the control than the PD group, albeit again the difference was not significant. vAm \% is a measure of the long-term variation in amplitude from any variations in the amplitude of the voice (periodic modulations, non-periodic modulation, rising or falling amplitude $)^{27}$. The study findings indicate therefore that vFo and vAm do not differentiate pwPD from their matched controls, and suggest that pwPD and controls have similar levels of overall unsteadiness in the voice, using acoustic measurement.

An important additional finding in this study which has implications for clinicians and researchers using MSP is that the control group values for Mftr\%, Matr \% vFo\% and vAm\% were higher than the CSL published norms ${ }^{27}$. These differences may be explained by a number of factors. Firstly, the participants were older in the current study than they were in the CSL sample. The mean (SD) age of the male and female combined group in this study was 60.11 (9.54); the mean (SD) age of the combined group published in the CSL manual was 37.9 (11.3) years. Age positively influences measures of frequency and amplitude modulation and overall variation. Secondly, CSL norms are based on speakers from the USA and it is unclear if these are representative of other cultures and nationalities. Thirdly, there were methodological differences which may explain the different findings for controls. In the current study, the mean value of three trials was used in the analysis and the middle 3 seconds was selected for analysis. For CSL norms, two trials were obtained but it is not reported if the mean value of the trials was used and/or if the middle three seconds was included ${ }^{27}$. Therefore, caution should be exercised when interpreting results generated from the MSP voice and tremor protocol for older patients.

Given the variability of populations examined, parameters measured, algorithms employed and methods for calculation of variables used in previous studies, it was not possible to 
arrive at a reliable a priori estimation of effect sizes that might exist between people with and without PD. To aid the interpretation of our findings and provide guidelines for future studies we conducted post hoc power analyses based on effect sizes found. In the context of some of the differences found between groups being small and statistically non-significant, coupled with large degrees of variability within groups, it was unsurprising that some of the resultant power estimations wereas small and indicated the need for much larger group sizes if statistically significant differences were to be sought. This was true in particular for Mftr\%, $\mathrm{vF0} \%$ and vAm\%. However, for other measures this study was sufficiently powered, adding to the conclusion that the differences observed were valid and reliable differences. This was true in particular for Rftr Hz, Ratr Hz, and Patr\%. Matr \% still attained a power of approximately 0.70, whereasitst Pftr\% was somewhat lower (approaching 0.6). Whilst power analyses are necessary to rule out or minimise type 1 and II errors, it is also useful to view outcomes in the context of clinical utility.

Based on these findings one can infer that Rftr $\mathrm{Hz}$, Ratr $\mathrm{Hz}$, Patr\% represent good candidate variables for the differentiation of normal age related tremor from pathological tremor, with Matr \% and Pftr\% not to be ignored. They should therefore form the core of future investigations into the topic. By contrast the remaining variables appear not to be reliable differentiators. Even if a study were to have sufficient participants to be adequately powered to detect a difference in the variables that showed small effect sizes, the clinical utility of those differences would be arguably low.

The results show that the selected acoustic tremor measures were not predictive of the total $\mathrm{VHI}$, or the VHI subscale scores, for pwPD. The finding that there was no relationship identified between acoustic tremor measures, and voice disability is not surprising. It is difficult to relate objective acoustic measures to the self-perceived impact of a voice disorder which varies for each patient depending on their personality, social networks, family relationships and occupation ${ }^{32}$. Although a number of studies have used acoustic, and selfreport measures 334532 in voice evaluation of pwPD, no previous study has explored the relationship between acoustic voice tremor and self-reported voice disability. The current findings highlight the nonlinear relationship that exists between impairment and disability ${ }^{34}$. Acoustic tremor measures and self-report voice disability measures are not interchangeable therefore based on the current results.

An important finding is that this group of pwPD were found to have a significantly greater voice disability than an age and sex-matched control group. Self-perceived voice problems in pwPD have been reported in other studies, some of which have also used the Voice Handicap Index $(\mathrm{VHI})^{33} 45$. The total VHI score for pwPD in the current study indicated a mild 
disability and was similar to the values reported by Frost et al. ${ }^{4}$ Conversely, Carmichael et al., ${ }^{33}$ in their study reported higher mean VHI scores [(mean 39.99,(SD) 22.35)] than those in the current study which may be explained by the fact that their group had a greater disease severity profile than pwPD in the current study. There was a wide range of total VHI scores in the PD group (0-49), with some participants showing little or no voice related disability. This is plausible since participants were not selected for the study based on auditory perceived speech or voice difficulties.

This preliminary work exploring voice tremor in the context of PD disease duration raises some interesting findings and questions. Results show that there was a significant positive correlation between magnitude of frequency tremor Mftr\% and disease duration in the current cohort. Therefore, as the length of time from diagnosis increased there was a corresponding increase in the magnitude of tremor. This may be revealing of the pathophysiology of PD voice tremor. For example, the fact that Mftr\% showed a stronger correlation with disease duration relative to Matr \%, may reflect that changes in the physiology of tremor over time impact more on the length of the vibratory cycle than on the amplitude. This is a preliminary supposition based on findings from a small sample of pwPD with a relatively short disease duration.

UPDRS II is used widely clinically to document the impact of the disease on the person's activities of daily living. Speech and voice studies do not generally report data on UPDRS II, therefore the findings here are novel and exploratory. Acoustic voice tremor was found not to relate positively or negatively to activities of daily living measured with UPDRS part II. Possible reasons for the lack of a relationship between the two measures are firstly, the level of disability as measured with UPDRS II may have been too low (mild) for any meaningful relationships to emerge. Secondly, computerised acoustic voice analysis was carried out at a discrete point in time whereas global disability related to PD was measured on the basis of the pwPD self-perception of specific symptoms and activities (tremor, speech, handwriting etc.) over the preceding two week period. Item 5, which is the speech item in UPDRS II relates to pwPD self-perception of speech intelligibility only and not to voice tremor or other speech/voice variables specifically. Finally, UPDRS II is a composite measure of pwPD selfreporting on different aspects of PD (walking, falling, tremor, handwriting etc) and may not relate in any way to voice tremor.

The relationship between acoustic voice tremor and motor symptom severity (UPDRS III) showed mixed findings. As PD motor symptoms become more pronounced, there is a lowering of the rate of amplitude tremor (Ratr $\mathrm{Hz}$ ). Neither the magnitude of tremor (frequency, amplitude) nor the overall variation of frequency or amplitude related in any way 
to UPDRS III scores. The findings suggest that as the disease process develops (increase in motor symptoms), the rate of tremor (amplitude) becomes slower. The rate of amplitude tremor differentiated pwPD from controls which strengthens the finding that Ratr and UPDRS are related at least in this group of pwPD. Pathological tremor is associated with a lower rate of tremor than normal 'physiological tremor'. One could speculate therefore, that the pathophysiology of voice tremor changes over the course of the disease leading to a lowering of the rate which translates into it being more noticeable clinically in more advanced stages of the disease.

\section{Conclusion}

The findings show that there are similarities and differences between pwPD and healthy controls when voice tremor is measured acoustically. Voice tremor is a feature of pwPD and controls. To differentiate pwPD from controls, the rate of amplitude tremor $(\mathrm{Hz})$ appears to be the most useful acoustic measure. Voice tremor increases in magnitude as the length of time from PD diagnosis increases. Although pwPD had significantly greater voice related disability than controls, acoustic voice tremor was not a contributory factor. As PD motor symptoms increase, the rate of amplitude tremor lowers. Oscillatory movement in the vocal tract in pwPD appears to impact more on the amplitude of the cycle than it does on the length of the cycle in this group of pwPD. Voice tremor analysis in PD contributes to increased understanding of phonatory dysfunction and its relationship with disease symptomatology. Acoustic analysis of PD voice tremor as conducted in this study enhances our understanding of the characteristics of voice tremor in PwPD in terms of rate, periodicity and magnitude of frequency and amplitude components. Further studies are required using additional measurement tools to determine the source of voice tremor i.e which structure/s in the vocal tract are moving involuntarily during phonation and resulting in auditory perceived tremor.

\section{Acknowledgements}

This study was completed as part of a doctorate by the first author who acknowledges the support of Speech \& Language Therapy, Neurology and Otolaryngology departments. A small research grant was gratefully received from the post-graduate college of the Mater Hospital. 


\section{APPENDIX 1}

\section{Tremor-related measures from the Voice and Tremor Protocol from Motor Speech Profile (MSP) Model 5141 of the Computerised Speech Laboratory (CSL)}

1. Rate of frequency tremor (Rftr) measured in $\mathrm{Hz}$ The frequency of the most intensive low-frequency Fo-modulating component in the specified Fo-tremor analysis range

2. Rate of amplitude tremor (Ratr) measured in $\mathrm{Hz}$

The frequency of the most intensive low-frequency amplitude-modulating component, in the specified amplitude-tremor analysis range

3. Periodicity of the frequency tremor [Pftr (\%)]

The periodicity of the frequency tremor. If the rate of tremor is very consistent, then it is a periodic tremor

4. Periodicity of the amplitude tremor [Patr(\%])

The periodicity of the amplitude tremor. If the rate of tremor is very consistent, then it is a periodic tremor

5. Magnitude of frequency tremor (Mftr), measured in \%

The magnitude or the extent of frequency tremor which can be from periodic or nonperiodic modulations

6. Magnitude of amplitude tremor (Matr), measured in \%

The magnitude or the extent of amplitude tremor which can be from periodic or from non- periodic modulations

7. Coefficient of variations in the Fundamental Frequency ( $\mathrm{vFo}$ ), measured in $\%$ $\mathrm{vFo}$ is the long-term variation in fundamental frequency $(\mathrm{Fo})$ from any variations in the Fo of the voice. Fo variations include periodic modulations, non-periodic modulations, and rising or falling Fo across the recorded segment

8. Coefficient of variations in the amplitude (vAm) measured in \%

$v A m$ is the long-term variation in amplitude from any variations in the amplitudes of the voice. Amplitude variations include periodic modulations, non-periodic modulations, and rising or falling amplitude 


\section{References}

1. Darley F, Aronson A, Brown J. Clusters of deviant speech dimensions in the dysarthrias. Journal of Speech \& Hearing Research 1969;12:462-96.

2. Ramig L, Bonitati C, Lemke J, Horii Y. Voice therapy for patients with Parkinson disease: development of an approach and preliminary efficacy data. Journal of Medical Speech-Language Pathology 1994;2:191-210.

3. Blumin J, Pcolinsky D, Atkins J. Laryngeal findings in Advanced Parkinson's Disease. Annals Otolaryngology Rhinology Laryngology 2004;113:253-58.

4. Frost E, Tripoliti E, Hariz M, Pring T, Limousin P. Self-perception of speech changes in patients with Parkinson's disease following deep brain stimulation of the subthalmic nucleus. International Journal of Speech-Language Pathology 2010;12(5):399-404.

5. Midi I, Dogan M, Koseoglu M, Can G, Sehitoglu M, Gunal D. Voice abnormalities and their relation with motor dysfunction in Parkinson's disease. Acta Neurological Scandinavia 2008;117:26-34

6. Dromey C, Reese L, Hopkin A. Laryngeal-level amplitude modulation in vibrato. Journal of Voice 2009;23(2):156-63.

7. Brin M, Fahn S, Blitzer A, Ramig L, Stewart C. Movement Disorders of the Larynx. Neurologic Disorders of the Larynx: Thieme, 1992.

8. Logemann J, Fisher H, Boshes B, Blonsky R. Frequency \& co-occurrence of vocal tract dysfunctions in the speech of large sample of Parkinson patients. Journal of Speech \& Hearing Disorders 1978;43:47-57.

9. Chenery J, Murdoch B, Ingram J. Studies in Parkinson's Disease: 1. Perceptual speech analyses. Australian Journal of Human Communication Disorders 1988;16:17-29.

10. Gamboa J, Jimenez-Jimenez F, Nieto A, Montojo J, Orti-Pareja M, Molino J, et al. Acoustic Voice Analysis in Patients with Parkinson's Disease Treated with Dopaminergic Drugs. Journal of Voice 1997;11(3):314-20.

11. Perez K, Ramig L, Smith M, Dromey C. The Parkinson Larynx: Tremor and Videostroboscopic Findings. Journal of Voice 1996;10(4):354-61.

12. Ramig L, Scherer R, Titze I, Ringel S. Acoustic Analysis of Voices of Patients with Neurological Disease: rationale and preliminary data. Annals Otolaryngology Rhinology Laryngology 1988;97:164-72.

13. Murdoch B, Manning C, Theodoros D, Thompson E. Laryngeal and phonatory function in Parkinson's disease. Clinical Linguistics and Phonetics 1997;11(3):245-56.

14. Boutsen F, Duffy J, Aronson A. Flutter or Tremor in Hypokinetic Dysarthria: A case study. In: Cannito M, Yorkston K, Beukelman D, editors. Neuromotor Speech Disorders. Baltimore: Paul H. Brookes, 1998:157-65.

15. Jimenez-Jimenez F, Gamboa J, Nieto A, Guerrero J, Orti-Pareja M, Molina J, et al. Acoustic Voice Analysis in Untreated Patients with Parkinson's Disease. Parkinsonism \& Related Disorders 1997;3(2):111-16.

16. Ludlow C, Bassich C, Connor N, Coulter D. Phonatory characteristics of vocal fold tremor. Journal of Phonetics 1986;14:509-15.

17. Jiang J, Lin E, Hanson D. Acoustic and Airflow Spectral Analysis of Voice Tremor. Journal of Speech, Language \& Hearing Research 2000;43:191-204.

18. Stewart C, Winfield L, Hunt A, Bressman S, Fahn S, Blitzer A, et al. Speech Dysfunction in Early Parkinson's Disease. Movement Disorders 1995;10(5):562-65.

19. Zarzur A, Duarte I, Goncalves G, Martins M. Laryngeal Electromyography and Acoustic Voice Analysis in Parkinsons Disease: a comparative status. Brazilian Journal Otorhinolaryngology 2010;76(1):40-3.

20. Tanaka Y, Nishio M, Niimi S. Vocal Acoustic Characteristics of Patients with Parkinson's Disease. Folia Phoniatrica et Logopaedica 2011;63(5):2011. 
21. Fahn S, Elton R. Unified Parkinson's Disease Rating Scale In: Fahn S, Goldstein M, D M, Calne D, editors. Recent Developments in Parkinson's Disease. New Jersey: Macmillan Healthcare Information, 1987:153-63.

22. Folstein M, Folstein S, Mc Hugh P. Mini-mental state. A practical method for grading the cognitive state of patients for the clinician. Journal of Psychiatric Research 1975;12:189-98.

23. Langston J, Widner H, Goetz C. Core Assessment Program for Intracerebral Transplantations. Movement Disorders 1992;7:2-13.

24. Jacobsen B, Johnson A, Grywalski C, Silbergleit A, Jacobsen G, Beninger M. The Voice Handicap Index (VHI): Development and Validation. American Jounal of Speech \& Language Pathology 1997;6:66-70.

25. Oguz H, Tunc T, Safak M, Inan L, Kargin S, Demirci M. Objective Voice Changes in Nondysphonic Parkinson's Disease Patients. Journal of Otolaryngology 2006;35(5):349-54.

26. Kent RD, J. Weismer, G. Ataxic Dysarthria. . Ataxic Dysarthria. Journal of Speech, Language \& Hearing Research 2000;43:1275-89.

27. Deliyski D, De Lassus Gress C. Software Instruction Manual: Motor Speech Profile (MSP) Model 5141. NJ: Kay Pentax, 2007.

28. Goberman A. Correlation between acoustic speech characteristics and non-speech motor performance in Parkinson Disease. Medical Science Monitor 2005;11:109-16.

29. D'Alatri L, Paludetti G, Contarino M, Galla S, Marchese M, Bentivoglio A. Effects of Bilateral Subthalamic Nucleus Stimulation and Medication on Parkinsonian Speech Impairment. Journal of Voice 2008;22(2):365-72.

30. Boutsen F, Duffy J, Dimassi H, Christman S. Long-Term Phonatory Instability in Ataxic Dysarthria. Folia Phoniatrica et Logopaedica 2011;63:216-20.

31. Kraus P, Lemke M. Kinetic tremor in Parkinson's disease - an underrated symptom. Journal of Neural Transmission 2006;113:845-53.

32. Wheeler K, Collins S, Sapienza C. The Relationship between VHI scores and specific acoustic measures of mildly disordered voice production. Journal of Voice 2006;20(2):308-17.

33. Carmichael C, Ruddy B. Respiratory Function \& Self Perceived Voice Handicap in Patient's with Parkinson's Disease. Texas Journal of Audiology \& Speech Language Pathology 2009;32:35-45.

34. Bornman J. The World Health Organisation's terminology and classifcation: application to severe disability. Disability and Rehabilitation 2004;26(3):182-88. 